\title{
Validity of a self-reported questionnaire for periodontitis in a Spanish population
}

\author{
Constanza Saka-Herrán $^{1}$ ｜ Enric Jané-Salas ${ }^{2}$ | Beatriz González-Navarro ${ }^{2}$ \\ Albert Estrugo-Devesa $^{2}$ | José López-López ${ }^{3}$
}

${ }^{1}$ Faculty of Medicine and Health Sciences (Dentistry), University of Barcelona, Barcelona, Spain

${ }^{2}$ Department of Odontostomatology, Faculty of Medicine and Health Sciences (Dentistry), University of Barcelona-Oral Health and Masticatory System Group (Bellvitge Biomedical Research Institute) IDIBELL, University of Barcelona, Barcelona, Spain

${ }^{3}$ Department of Odontostomatology, Faculty of Medicine and Health Sciences (Dentistry), Odontological Hospital University of Barcelona, University of Barcelona Oral Health and Masticatory System Group (Bellvitge Biomedical Research Institute) IDIBELL, University of Barcelona,

Barcelona, Spain

\section{Correspondence}

José López-López, University Campus of Bellvitge, Pabellón de Gobierno, 2nd floor, office 2:29-Feixa Llarga, s/n, 08907-L' Hospitalet de Llobregat, Barcelona, Spain. Email:18575j11@gmail.com

\begin{abstract}
Background: Clinical examination is the gold-standard approach for surveillance of periodontitis; however, it requires large resources. Several self-reported measures have been developed and tested in diverse scenarios with results suggesting that it may be a useful tool for screening periodontal disease in different populations; yet they have not being tested in Spanish population. We aimed to assess the validity of a self-reported questionnaire for periodontitis in a Spanish population from Barcelona during 2018.
\end{abstract}

Methods: One hundred and twelve participants were enrolled in the study and, in one appointment; a dentist performed the self-reported questionnaire and a fullmouth periodontal examination. Periodontitis was defined as at least mild periodontitis according to three criterion of classification. Receiver-operating characteristics curve analyses were used to test the discriminatory capability, sensitivity and specificity of the self-reported questionnaire and logistic regression models were adjusted to estimate the minimal-set of questions associated with periodontitis.

Results: The self-reported questionnaire had a useful discriminative capability for detecting individuals with periodontitis (area under the curve [AUC] $=0.8595 \% \mathrm{CI}$ 0.78-0.92) and its moderate/severe form (AUC $=0.8695 \%$ CI 0.79-0.04) with sensitivity and specificity of $77 \%$ and $74 \%$ and $73 \%$ and $87 \%$, respectively; representing moderate validity. The combination of four-specific questions had high accuracy (AUC $=0.8895 \%$ CI 0.81-0.94) and validity (sensitivity $=92.2 \%$ ) and was strongly associated with moderate/severe periodontitis after adjusting by socio-demographic factors.

Conclusions: The use of several self-reported questions proved to have a good performance for screening periodontitis in the population under study; specifically, those related with tooth mobility and gum migration. Large community-based studies are needed to test its validity and predictive capability.

\section{K E Y W O R D S}

dental health surveys, periodontal diseases, periodontitis, self report, surveys and questionnaires 


\section{1 | INTRODUCTION}

Periodontal disease is a multifactorial chronic inflammatory disease, which is estimated to affect half of adults and its moderate form, one third of the population. ${ }^{1}$ In 2010 , severe periodontitis affected 734 million people worldwide, being the sixth most prevalent condition $(10.8 \%){ }^{2}$ In Spain, the prevalence of clinical signs of periodontitis was $80.6 \%$ in adults between 35 and 44 years and $89 \%$ in those between 64 and 75 years. ${ }^{3}$ Because of the large number of affected individuals and its high burden of disease, it is important that periodontitis be monitored at different levels of the population and over time, to evaluate the development and implementation of preventive or therapeutic interventions in the control and progression of this disease.

Currently, clinical examination is the standard and preferred approach for surveillance of periodontitis, ${ }^{4}$ which represents the gold standard. However, quantifying periodontal disease in a meaningful and reproducible manner has been of great challenge for both oral clinicians and epidemiologists. ${ }^{2}$ This, because of the different classifications systems that have been developed to describe the clinical manifestations of periodontitis, which differs in case definitions and scales for quantifying its severity. ${ }^{2}$ In addition, its implementation requires a large number of resources and, usually, is expensive, time-demanding ${ }^{5}$ and needs specialized professionals All this means that it cannot be easily implemented as a surveillance system in many countries, regions and at different levels of the population. ${ }^{6}$ The existence and use of a valid, low-cost and low-resources self-reported measures for periodontitis would be favorable in a variety of ways. It would facilitate epidemiological studies on a much larger scale that is feasible with clinical measures, would be an easier and lowcost method of obtaining data for research and surveillance of the periodontal status of populations over time. ${ }^{6}$

The validity of self-reported questionnaires differs between studies, populations and in their outcomes. Slade et al. ${ }^{7}$ reported $58 \%$ sensitivity and $81 \%$ specificity for a six-item questionnaire combined with known risk factors for periodontal disease in the prediction of moderate/severe periodontitis in 2999 Australians over 15 years. Eke et al., in a representative American population, assessed the predictive performance of eight self-reported questions against clinically classified periodontitis. The best performance in the prediction of periodontitis was the combination of demographic measures and responses to five self-reported questions with $85 \%$ sensitivity and $58 \%$ specificity. When periodontitis was defined as clinical attachment loss (CAL) $>3 \mathrm{~mm}$, sensitivity and specificity were $93.8 \%$ and $30.8 \%$, respectively, and when the probing depth (PD) $\geq 4 \mathrm{~mm}$ criterion was used values obtained were $76.1 \%$ and $58.4 \%$, respectively, concluding that self-reported measures performed well in predicting periodontal disease in US adults. In 288 Jordanian adults, Khader et al. ${ }^{8}$ tested the predictive validity of a six-item self-reported questionnaire for periodontitis against sev en different definitions of periodontal disease. Receiver-operating characteristics (ROC) curve analyses varied between 0.77-0.83, showing a useful performance of the questionnaire, regardless of the clinical definition used. Likewise, the sensitivity ranged between $61 \%$ and $83 \%$ and the specificity between $68 \%$ and $83 \%$, according to the clinical classification used. Zhan et al. ${ }^{5}$ developed a model for predicting moderate/severe periodontitis using self-reported questions and validated it in a different German population against three clinical classifications of periodontitis. The predictive model in the SHIP-0 population included demographic variables and two self-reported questions for periodontitis. The validation of the model in the SHIP-Trend population showed sensitivity between $73.5 \%$ and $82.6 \%$ and specificity between $72.6 \%$ and $74.9 \%$ in the prediction of moderate/severe periodontitis.

These self-reported measures have not been validated in Spain and, to our knowledge; no specific self-reported questionnaire for surveillance of periodontitis exists for Spanish population. We developed a self-reported questionnaire in Spanish considering as reference the eight self-reported oral questions from Eke et al. ${ }^{4}$ and developed some questions that, in our judgment, would be suitable indicators for screening periodontal disease in the population. Our main objective was to assess the validity and test the performance of this self-reported questionnaire in a Catalonian adult population during 2018.

\section{2 | METHODS}

A prospective diagnostic study was conducted. The study population were adults from the VI District (BellvitgeGornal) of Hospitalet de Llobregat geographic location and patients who attended the Dental Hospital from University of Barcelona (HOUB), Faculty of Medicine and Health Sciences (Dentistry), Bellvitge campus, during March-December 2018. Inclusion criteria were adult's $\geq 18$ years, who were capable to understand and respond to a self-reported questionnaire and who gave their informed consent to participate in the study. Exclusion criteria were totally edentulous adults or with $<10$ teeth and those with complete rehabilitation on osseointegrated implants. Eligible patients from the HOUB were identified through their first visit for dental review and participants from the general population through the neighborhood of Bellvitge's Hospital and were invited to participate if they met the inclusion criteria. We considered the results from our pilot study in 63 patients to compute sample size. The prevalence of periodontitis was $43 \%$ and the sensitivity of $78 \%$, which was established as the alternative hypothesis. Null hypothesis was considered as $60 \%$ based on the results 
from previous studies that reported 58\% sensitivity ${ }^{7}$ and $59 \%$ sensitivity ${ }^{4}$ for their self-reported questionnaires. With a $5 \%$ of significance level and $80 \%$ of power, the sample size was estimated in 45 participants with positive disease and a total sample size of 112 participants $^{9}$; 56 adults from the general population and 56 adults who attended the HOUB, meeting all the same eligibility criteria. The Clinical Research Ethics Committee from the HOUB approved the study on December 2017 (ID: 2017-44) and was conducted in accordance with the Helsinki Declaration, as revised in 2013. All participants gave written informed consent.

Participants were consecutively invited to participate and enrolled in the study. Each participant was scheduled on a specific day to the HOUB, were the study was conducted. The self-reported questionnaire was given to participants on the scheduled visit before periodontal examination was performed. It was a self-reading and self-answered questionnaire. In the same appointment, participants responded to a general-health questionnaire, the self-reported questionnaire and a calibrated dentist performed a full-mouth periodontal examination complemented with an orthopantomography, in that order. Length-time from the self-reported questionnaire to the clinical examination was $<10$ minutes. The dentist was blind to participants' answers to the self-reported questionnaire and participants were blinded to the results of the periodontal examination. Periodontal examination was done with a periodontal probe $(\mathrm{CP} 12)$ recording $\mathrm{CAL}$, depth on probing, presence/absence of calculus and bleeding on probing in six sides (mesio-vestibular, mid-vestibular, disto-vestibular, mesio-lingual/palatine, mid-lingual/palatine, disto-lingual/palatine) of all teeth and registered in a periodontal chart. Remaining teeth and plaque index (O'Leary Index $)^{10}$ was also recorded. Periodontitis classification was performed according to the Spanish Society of Periodontics and Osseointegration (SEPA), ${ }^{11}$ the International Workshop for a Classification of Periodontal Disease and Conditions (IWCPDC $)^{12}$ and the Centres for Disease Control and Prevention and the American Academy of Periodontology (CDCAAP) Working Group. ${ }^{13}$ The self-reported questionnaire was a 13-item questionnaire (one general and 12 specific questions) with a yes/no answer alternative (Table 2). The general question intended to assess the awareness of the periodontal conditions of participants.

Case definitions of periodontitis were the gold standard and were classified as presence or absence of periodontitis and its severity as mild, moderate, or severe according to the three criterion of classification. The SEPA classification for periodontitis is depth on probing $\geq 4 \mathrm{~mm}$ in at least one sextant and/or grade 2 to 3 furcation defects. ${ }^{11}$ The severity of periodontitis according to the IWCPDC is primarily based on interdental CAL at the site of greatest loss and radiographic bone loss and is classified in: stage I (initial periodontitis): CAL 1 to $2 \mathrm{~mm}$, coronal third ( $<15 \%)$; stage II (moderate periodontitis): CAL 3 to $4 \mathrm{~mm}$, coronal third (15\% to 33\%); stage III (severe periodontitis): $\mathrm{CAL} \geq 5 \mathrm{~mm}$, extending to mid-third of root and beyond and stage IV (advanced periodontitis): CAL $\geq 8 \mathrm{~mm}$, extending to mid-third of root and beyond. ${ }^{12}$ The CDC-AAP criteria for classification is mild periodontitis: $\geq 2$ interproximal sites with $\geq 3 \mathrm{~mm}$ of CAL and $\geq 2 \mathrm{~mm}$ interproximal with $4 \geq \mathrm{mm}$ PD (not on the same tooth) or one site with $\geq 5 \mathrm{~mm}$, moderate periodontitis: $\geq 2$ interproximal sites with $\geq 4 \mathrm{~mm}$ CAL (not on the same tooth) or $\geq 2$ interproximal sites with $\mathrm{PD} \geq 5 \mathrm{~mm}$, also not on the same tooth and severe periodontitis: $\geq 2$ interproximal sites with $\geq 6 \mathrm{~mm}$ CAL (not on the same tooth) and $\geq 1$ interproximal site(s) with $\geq 5$ mm PD. ${ }^{13}$

Periodontitis was defined as at least mild periodontitis, combined mild, moderate, and severe. The variable total_periodontitis was created combining the cases of periodontitis according to the three classifications (total_ periodontitis: PD_SEPA $=$ yes or PD_IWCPDC $=$ yes or PD_CDC-AAP = yes) and the variable total_mod/sev_ periodontitis combining the cases of moderate and severe periodontitis according to the IWCPDC and CDC-AAP classifications (total_mod/sev_periodontitis: PD_IWCPDC = yes or PD_CDC-AAP = yes) and were used for ROC curves analyses and logistic regression models. Each question from the self-reported questionnaire was classified as a dichotomous variable (yes/no). Variables such as age (years), sex (female/male), educational level (without education, basic, superior education), monthly salary (low income (minimum salary $=736$ EUR/816 USD), middle income ( 2 to 4 minimum salary), high income ( $>4$ the minimum salary)), country of origin (Spanish, non-Spanish), smoking habit (never, ex, current smoker), diabetes (self-reported diagnoses by a health professional), active medication and dietetic habits through the healthy diet questionnaire adapted from the Spanish Society of Atherosclerosis which have 14 questions with a yes/no answer (unhealthy diet $=\leq 4$ points, regular diet $=5$ to 9 points, healthy diet $=10$ to 14 points $)^{14}$ were recorded to explore their influence on the multivariable models for periodontitis.

\section{1 | Statistical analyses}

Data were collected through the Excel program. ${ }^{*}$ Numerical variables were described according to their distribution in mean and standard deviation or median and range; categorical variables in frequency and percentage. Associations between each question and the clinical definitions of periodontitis were carried out thorough the chi-square test and crude odds ratio (OR). Logistic regression models were adjusted to estimate the most significant set of self-reported questions

\footnotetext{
* Microsoft Excel, Microsoft Corporation, Redmond, WA.
} 
associated with periodontitis and its degrees of severity (moderate/severe). ROC curves analyses were used to examine the discriminatory capability, sensitivity and specificity of the 12-item questions and the minimal significant set of questions of the self-reported measures against the variables total_periodontitis and total_mod/sev_periodontitis. The most significant set of questions were combined and logistic regression models were adjusted to estimate its association with periodontitis and its moderate/severe form as response variables, adjusting by age, sex, education level, monthly salary, country of origin and tobacco use. Also area under the curve (AUC) analyses, sensitivity and specificity through ROC curves were performed to estimate the validity and discriminative capability of the combined set of questions. Estimates were reported with $95 \%$ confidence intervals and a $P$-value $<0.05$ was considered statistically significant. All analyses were performed with SPSS Statistics.

\section{3 | RESULTS}

\section{1 | Participants' characteristics}

A total of 112 adults were included in the study. Supplementary Figure 1 shows the flow of participants through the study (see Supplementary Figure 1 in online Journal of Periodontology). The mean age of the total sample was $46 \pm 17.9$ years, $57 \%$ were woman and $80 \%$ were original from Spain. Half of participants had both a professional training or college education and a middle monthly income. In addition, $30.4 \%$ were current smokers and $43.7 \%$ have unhealthy diet. The prevalence of diabetes and high blood pressure was $4.5 \%$ and $17 \%$, respectively (Table 1A). Compared with patients from the HOUB, participants from the general population were younger, $70 \%$ were from Spain, have a higher level of education, were less never smokers and mostly had an unhealthy diet. They also have a lower prevalence of diabetes and hypertension (Table 1A).

\section{2 | Prevalence and severity of periodontal disease}

According to the three clinical classifications for periodontal disease, prevalence of periodontitis in the whole sample was $43.8 \%$ according to the SEPA classification and $45.5 \%$ according to the IWCPDC and CDC-AAP classifications. Most participants had moderate and severe periodontitis as shown in Table 1B. Adults from the general population have a

\footnotetext{
* IBM SPSS Statistics, IBM Corporation, Armonk, NY.
}

lower prevalence of periodontitis and a less severe form compared with patients from the HOUB (Table 1B).

\section{3 | Self-reported periodontal disease questionnaire}

The item response rate for all questions was $100 \%$. The most frequent positive response was to the question: do you think you might have gum disease? (Q2.1) (44.6\%); although 32 participants, from the 50 who have a positive response, had actually periodontitis (64\%) (Table 2). Almost all of the self-reported questions from the questionnaire were significantly associated with periodontitis, regardless of the clinical definition used. The questions have you felt pain in your gums during the last months? (Q2.7) and do your gums usually bleed either when brushing or chewing? (Q2.12) were not associated with periodontitis. The strongest crude associations were seen for: have you ever been diagnosed by a dental professional with periodontal disease or pyorrhea? (Q2.2), in the past years have you noticed that your teeth are longer or that you have receding gums? (Q2.5) and have you lost teeth in recent years because of mobility? (Q2.11) as shown in Table 2.

\section{4 | Performance of the self-reported questionnaire on periodontal disease}

For the three clinical classifications for periodontitis the questions with the higher sensitivity were, do you think you might have gum disease? (Q2.1) (63.3\%) $(P=0.001)$ and in the past years have you noticed that your teeth are longer or that you have receding gums? (Q2.5) (70\%) $(P<0.001)$, with the latter having better values of specificity (Table $3 \mathrm{~A}$ ). The calculated AUC for the specific self-reported questionnaire (12-item questions) for discriminating those with periodontitis was 0.85 (95\% CI $0.78-0.92$ ) with a $77 \%$ of sensitivity and $74 \%$ of specificity (see Supplementary Figure 2 in online Journal of Periodontology).

From the 12-item questions, three questions were significantly associated with total periodontitis (total_periodontitis: PD_SEPA $=$ yes or PD_IWCPDC $=$ yes or PD_CDC$\mathrm{AAP}=\mathrm{yes})$ : in the past years have you noticed that your teeth are longer or that you have receding gums? (Q2.5), have you lost teeth in recent years because of mobility? (Q2.11) and do your gums usually bleed either when brushing or chewing? (Q2.12) (Table 4). These questions were used and combined in multivariable modeling to estimate the best-reduced set of questions associated with periodontal disease. The combination of the three questions had a sensitivity of $90.2 \%$ and discriminative capability of 0.87 (AUC $=0.8795 \% \mathrm{CI}$ 0.81-0.94); and after adjusting by age, sex, level of education, monthly salary and country of origin, this set of questions was significantly associated with periodontitis $(\mathrm{OR}=15.495 \% \mathrm{CI}$ 
T A B L E 1 A) Demographic characteristics, habits and medical conditions of the sample. B) Prevalence of periodontitis according to the three clinical classifications of periodontal conditions $(n=112)$

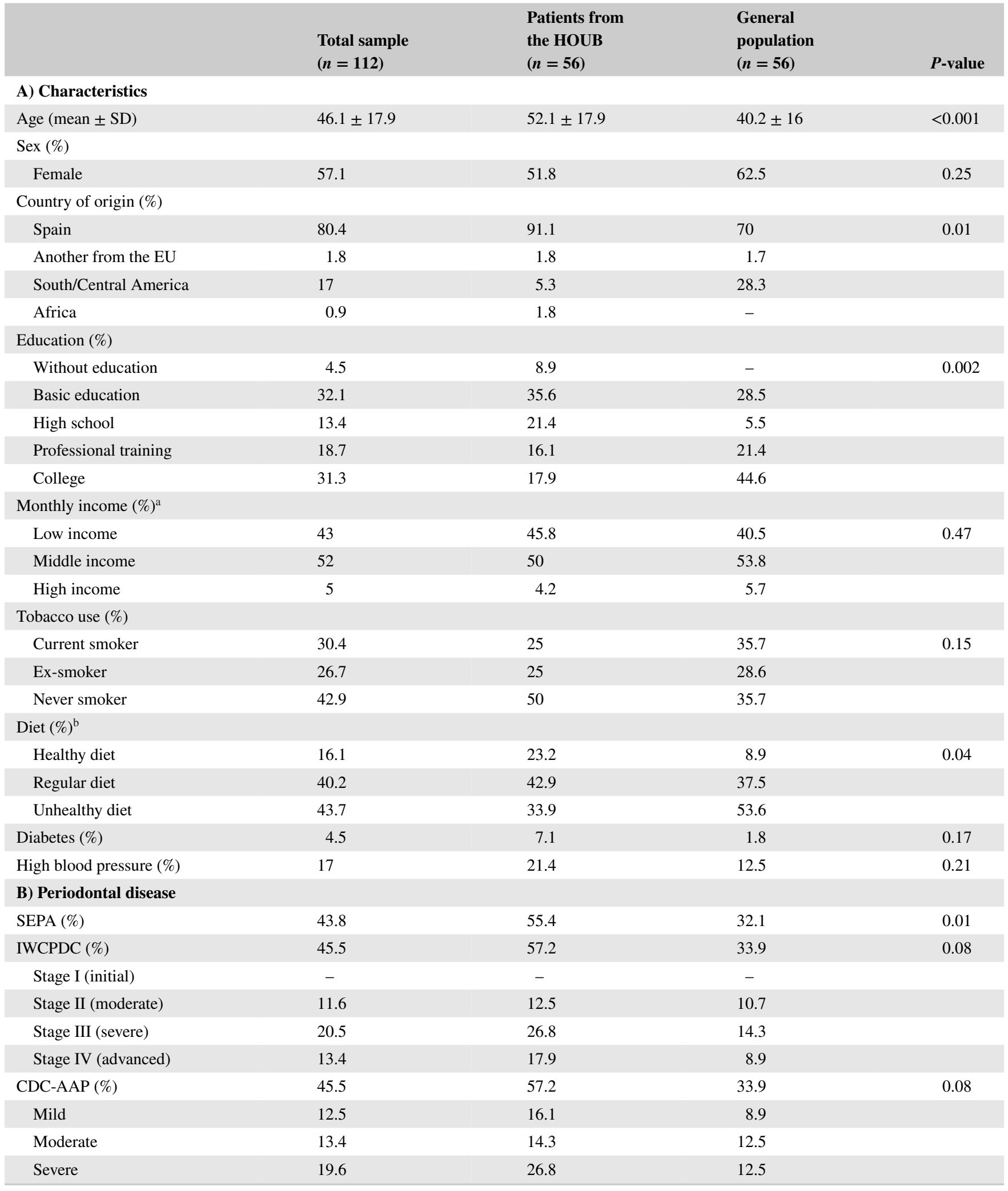

CDC-AAP, Centers for Disease Control and Prevention - American Academy of Periodontology Working Group; EU, European Union; HOUB, Dental Hospital from University of Barcelona; IWCPDC, International Workshop for a Classification of Periodontal Disease and Conditions; SEPA, Spanish Society of Periodontics and Osseointegration.

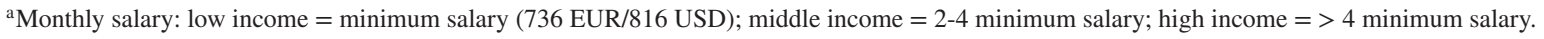

${ }^{\mathrm{b}}$ Diet: healthy diet questionnaire adapted from the Spanish Society of Atherosclerosis. ${ }^{14}$ 
T A B L E 2 Self-reported periodontal screening questionnaire, frequency of positive responses to the self-reported questions and crude associations according to the three clinical classifications of periodontal disease $(n=112)$

\begin{tabular}{|c|c|c|c|c|c|}
\hline Self-reported questions & $\begin{array}{l}\text { Response } \\
\text { (YES) } \\
n(\%)\end{array}$ & $\begin{array}{l}\text { TP } \\
n(\%)\end{array}$ & $\begin{array}{l}\text { SEPA } \\
\text { OR }(95 \% \mathrm{CI})\end{array}$ & $\begin{array}{l}\text { IWCPDC } \\
\text { OR }(95 \% \mathrm{CI})\end{array}$ & $\begin{array}{l}\text { CDC-AAP } \\
\text { OR }(95 \% \mathrm{CI})\end{array}$ \\
\hline Q_1 ¿Tiene usted enfermedad periodontal? & $32(28.6)$ & $20(62.5)$ & $2.4(1.1-5.6)$ & $2.6(1.1-6.1)$ & $2.6(1.1-6.1)$ \\
\hline \multicolumn{6}{|l|}{ Do you have periodontal disease? } \\
\hline $\begin{array}{l}\text { Q_2.1 ¿Piensa usted que tal vez tenga enfermedad de las } \\
\text { encías? }\end{array}$ & $50(44.6)$ & $32(64)$ & $3.9(1.8-8.8)$ & $4.0(1.8-8.9)$ & $4.0(1.8-8.9)$ \\
\hline \multicolumn{6}{|l|}{ Do you think you might have gum disease? } \\
\hline $\begin{array}{l}\text { Q_2.2 ¿Alguna vez algún dentista le ha diagnosticado } \\
\text { que tiene enfermedad periodontal o piorrea? }\end{array}$ & $21(18.8)$ & $20(95.2)$ & $42.7(5.5-334.2)$ & $38.7(4.9-302.1)$ & $38.7(4.9-302.1)$ \\
\hline
\end{tabular}

Have you ever been diagnosed by a dental professional with periodontal disease or pyorrhea?

Q_2.3 ¿Alguna vez algún dentista le ha dicho que ha $\quad 22(19.6) \quad 19(86.4) \quad 8.6(2.7-27.5) \quad 11.5(3.2-41.8) \quad 11.5(3.2-41.8)$ perdido hueso alrededor de los dientes o que tiene bolsas profundas?

Have you ever been told by a dental professional that you have lost bone around your teeth or that you have deep pockets?

Q_2.4 ¿En los últimos años ha notado que algunos de $\quad 33$ (29.5) $\quad 28$ (84.8) $\quad 11.6(4.2-32.1) \quad$ 13.6 (4.7-39.7) $\quad$ 13.6 (4.7-39.7) sus dientes se mueven o están mas sueltos de lo normal?

In the last years have you noticed that some of your teeth move or are looser than normal?

Q_2.5 ¿En los últimos años ha notado que sus dientes $\quad 42(37.5) \quad 36(85.7) \quad 15.6(5.9-40.6) \quad 22.0$ (7.8-61.9) $\quad 22.0$ (7.8-61.9) están más largos y/o las encías más arriba (retraídas) de lo normal?

In the past years have you noticed that your teeth are longer or that you have receding gums?

Q_2.6 ¿En los últimos años ha notado que se le ven las $\quad 24(21.4) \quad 19(79.2) \quad 5.5(1.9-15.3) \quad 6.7(2.3-19.5) \quad 6.7(2.3-19.5)$ raíces de varios de sus dientes?

In the last years have you noticed that you see the roots of several of your teeth?

Q_2.7 ¿En los últimos meses ha notado que le duelen $\quad 33(29.5) \quad 17(51.5) \quad 1.3(0.6-2.9) \quad 1.4(0.6-3.2) \quad 1.4(0.6-3.2)$ sus encías?

Have you felt pain in your gums during the last months?

Q_2.8 ¿Utiliza usted palillo o cepillo interproximal para limpiarse los dientes con frecuencia?

Do you frequently use a stick or interproximal brush to clean your teeth?

Q_2.9 ¿Alguna vez ha visitado a un periodoncista o $\quad 16(14.3) \quad 12$ (75) $\quad 3.4(1.1-10.4) \quad 4.4(1.3-14.6) \quad 4.4(1.3-14.6)$ especialista en encías para tratarse la enfermedad de las encías?

Have you ever visited a periodontist or a specialist in gum disease to treat gum disease?

Q_2.10 ¿Alguna vez ha tenido tratamiento de las encías $13(11.6) \quad 10$ (76.9) $\quad 5.1(1.3-19.8) \quad 4.7(1.2-18.2) \quad 4.7$ (1.2-18.2) tipo raspado o alisado de las raíces?

Have you ever had treatment for gum disease such as scaling or root planing?

Q_2.11 ¿Ha perdido dientes en los últimos años por $\quad 23(20.5) \quad 22(95.7) \quad 22.9(5.1-104.3) \quad 45.5(5.8-354.4) \quad 45.5(5.8-354.4)$ movilidad?

Have you lost teeth in recent years because of mobility?

Q_2.12 ¿Le sangran habitualmente las encías al cepillar o al masticar?

Do your gums usually bleed either when brushing or chewing?

CDC-AAP, Centers for Disease Control and Prevention - American Academy of Periodontology; IWCPDC, International Workshop for a Classification of Periodontal Disease and Conditions; OR, odds ratio; SEPA, Spanish Society of Periodontics and Osseointegration; TP, True positives. 


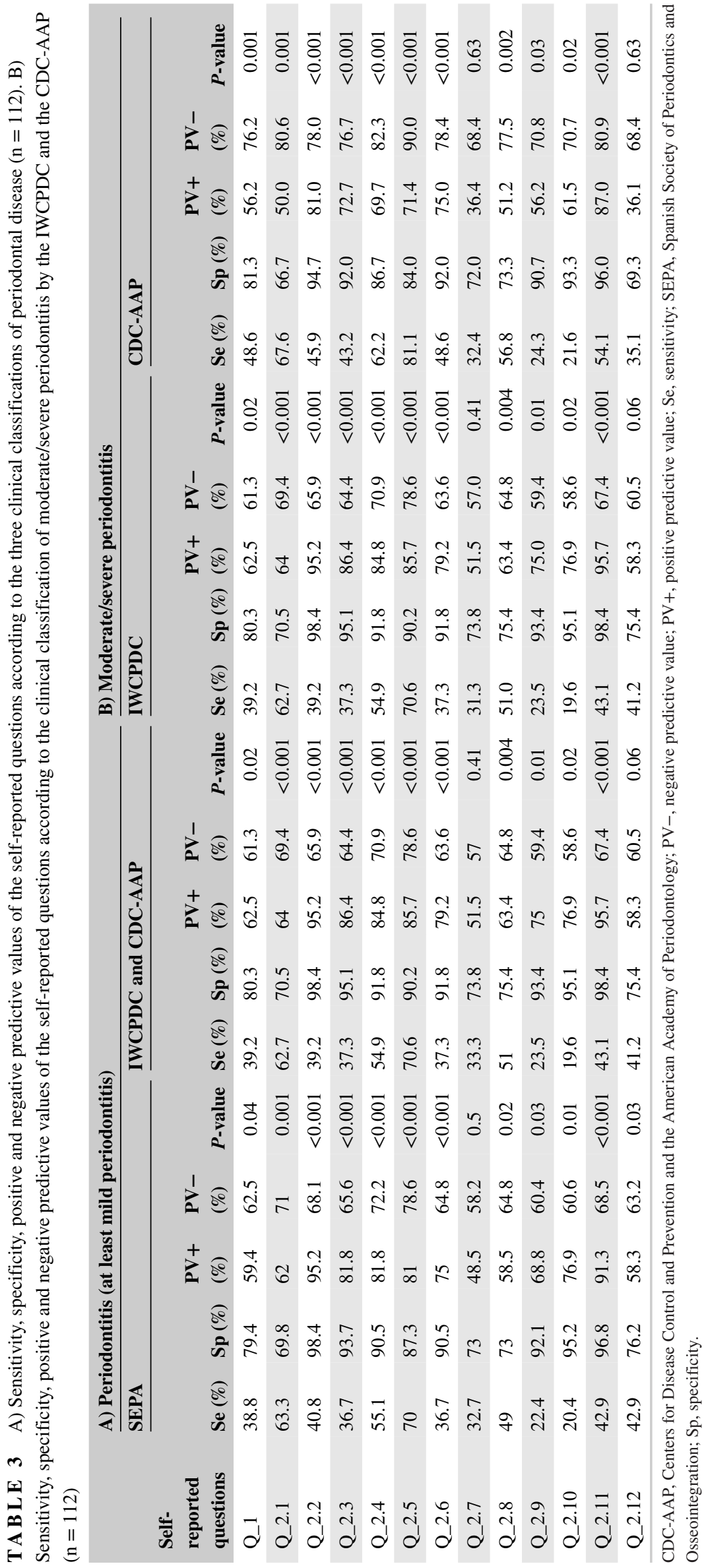


T A B L E 4 Logistic regression model to estimate the minimum significant set of questions of the self-reported questionnaire associated to total periodontitis and to its moderate/severe form $(n=112)$

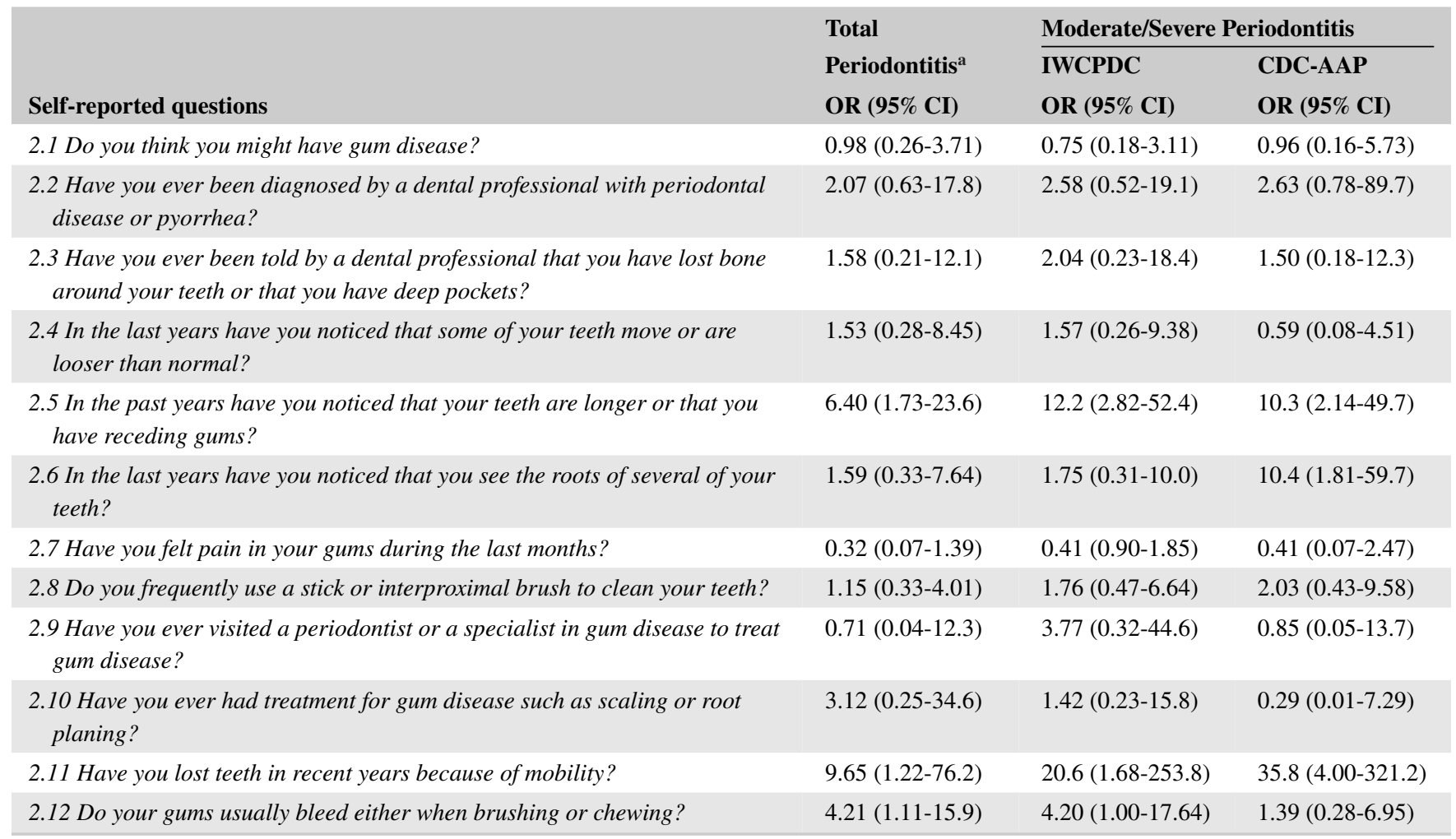

CDC-AAP, Centers for Disease Control and Prevention - American Academy of Periodontology; IWCPDC, International Workshop for a Classification of Periodontal Disease and Conditions; OR, odds ratio.

${ }^{\mathrm{a}}$ Response variable: periodontal disease $=$ YES $\left(\mathrm{PD} \_\mathrm{SEPA}=\right.$ yes or PD_IWCPDC $=$ yes or PD_CDC-AAP $=$ yes $)$.

T A B L E 5 Sensitivity, specificity, positive and negative predictive values and odds ratio for the most significant questions related to periodontal disease $(n=112)$

\begin{tabular}{|c|c|c|c|c|c|c|c|c|c|}
\hline $\begin{array}{l}\text { Self-reported } \\
\text { questions }\end{array}$ & $\begin{array}{l}\text { Response } \\
\text { (Yes) } \\
\text { n (\%) }\end{array}$ & $\begin{array}{l}\mathrm{Se} \\
(\%)\end{array}$ & $\begin{array}{l}\text { Sp } \\
(\%)\end{array}$ & $\begin{array}{l}\text { PV+ } \\
(\%)\end{array}$ & $\begin{array}{l}\text { PV- } \\
(\%)\end{array}$ & $P$-value & $\operatorname{AUC}(95 \% \mathrm{CI})$ & $\begin{array}{l}\text { Crude OR } \\
(95 \% \text { CI })\end{array}$ & $\begin{array}{l}\text { Adjusted OR } \\
(95 \% \text { CI })\end{array}$ \\
\hline $\begin{array}{l}2.5 \text { or } 2.11 \text { or } 2.12 \\
\quad \text { questions }\end{array}$ & $66(58.9)$ & 90.2 & 67.2 & 69.7 & 89.1 & $<0.001$ & $0.87(0.81-0.94)$ & $18.8(6.5-54.7)$ & $15.4(4.1-57.8)$ \\
\hline 2.5 or 2.11 questions & $49(43.8)$ & 80.4 & 86.9 & 83.7 & 84.1 & $<0.001$ & $0.86(0.79-0.94)$ & $27.1(9.8-74.9)$ & $21.2(6.1-73.9)$ \\
\hline 2.5 or 2.12 questions & $62(55.4)$ & 84.3 & 68.9 & 69.4 & 84.0 & $<0.001$ & $0.79(0.71-0.88)$ & $11.9(4.7-30.1)$ & $15.2(4.1-57.0)$ \\
\hline 2.11 or 2.12 questions & $50(44.6)$ & 66.7 & 73.8 & 68.0 & 72.6 & $<0.001$ & $0.73(0.63-0.82)$ & $5.6(2.5-12.7)$ & $6.27(2.1-19.9)$ \\
\hline 2.1 or 2.5 questions ${ }^{\mathrm{b}}$ & $62(55.4)$ & 82.4 & 67.2 & 67.7 & 82.0 & $<0.001$ & $0.80(0.72-0.89)$ & $9.6(3.9-23.4)$ & $17.0(4.2-68.4)$ \\
\hline
\end{tabular}

AUC, area under the curve; OR, odds ratio; PV-, negative predictive value; $\mathrm{PV}+$, positive predictive value; Se, sensitivity; Sp, specificity.

Response variable: periodontal disease $=$ YES $($ PD_SEPA $=$ yes or PD_IWCPDC $=$ yes or PD_CDC-AAP $=$ yes).

${ }^{a}$ Adjusted by age, sex, education, monthly income, smoking status and country of origin.

${ }^{\mathrm{b}}$ Adjusted model for most sensitive questions related to periodontitis.

4.1-57.8). Also, the combination of two questions: in the past years have you noticed that your teeth are longer or that you have receding gums? (Q2.5) and have you lost teeth in recent years because of mobility? (Q2.11) had a $80.4 \%$ of sensitivity, $87 \%$ of specificity, $83.7 \%$ positive predictive value and $84.1 \%$ negative predictive value. The adjusted model was also significantly associated with periodontitis $(\mathrm{OR}=21.295 \% \mathrm{CI}$ 6.1-73.9) (Table 5).

\section{5 | Performance of the self-reported questionnaire on moderate to severe periodontitis}

As with total periodontitis, the most sensitive questions for moderate/severe periodontitis according to the IWCPDC and the CDC-AAP Working Group classifications were do you think you might have gum disease? (Q2.1) and in the past years have you noticed that your teeth are longer or that 
T A B L E 6 Sensitivity, specificity, positive and negative predictive values and odds ratio for the most significant questions related to moderate/severe periodontitis $(\mathrm{n}=112)$

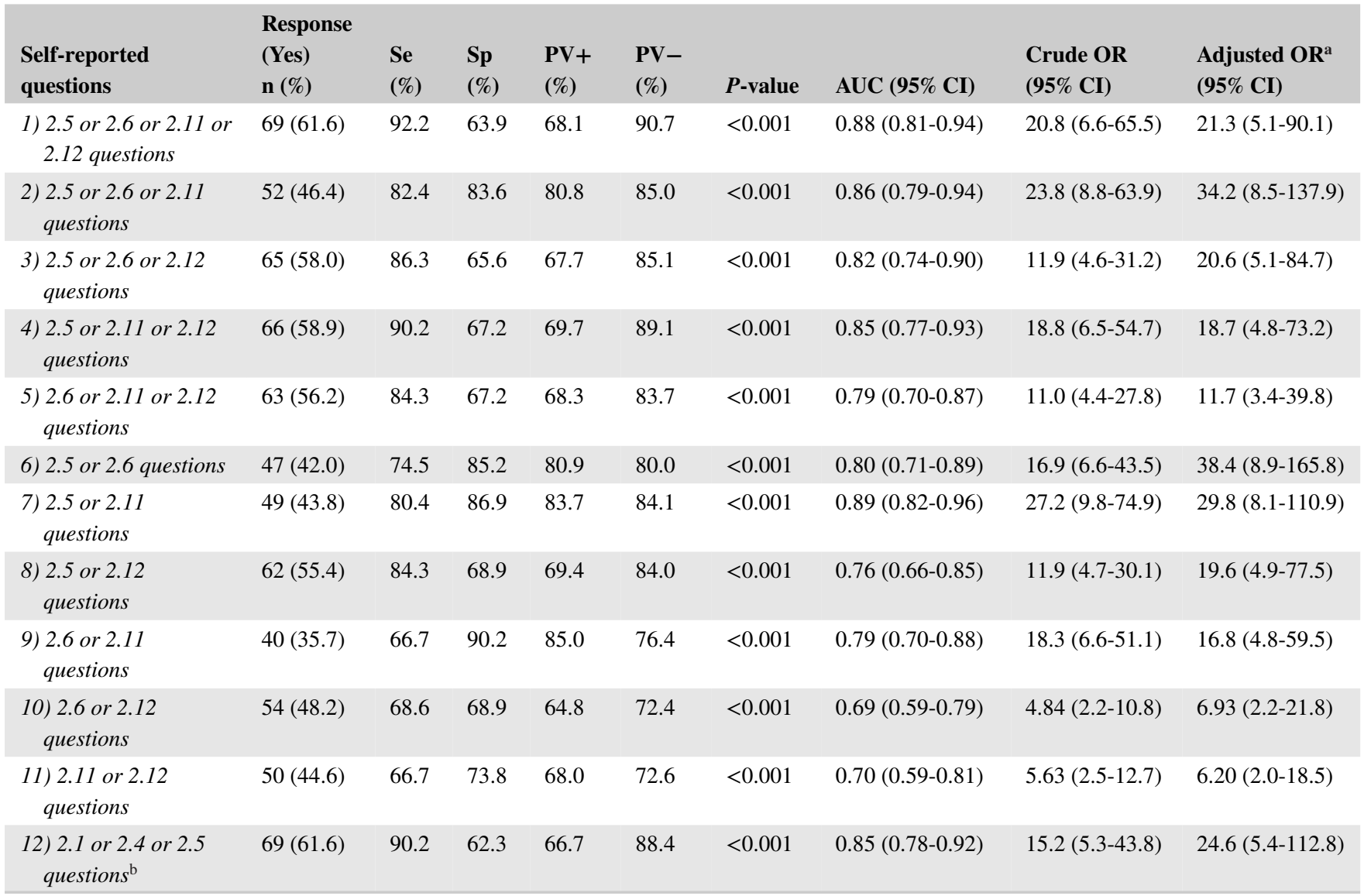

AUC $<$ area under the curve; OR, odds ratio; PV-, negative predictive value; PV+, positive predictive value; Se, sensitivity; Sp, specificity.

Response variable: moderate/severe periodontitis $=$ YES (IWCPDC_mod/sev $=$ yes or CDC-AAP_mod/sev = yes).

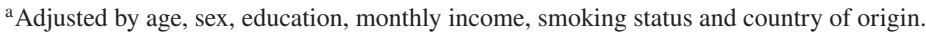

${ }^{\mathrm{b}}$ Adjusted model for most sensitive questions related to moderate/sever periodontitis.

you have receding gums? (Q2.5); the latter with greater values of specificity. Additionally, the question in the last years have you noticed that some of your teeth move or are looser than normal? (Q2.4) had a higher sensitivity according to the CDC-AAP classification (Table 3B). According to both classifications, the 12-item questionnaire had a useful accuracy for discriminating those with moderate/severe periodontitis with AUC values of 0.85 (95\% CI 0.78-0.92) and 0.86 (95\% CI 0.79-0.94) (see Supplementary Figure 3.1 and 3.2 in online Journal of Periodontology).

From the adjusted model, both questions In the past years have you noticed that your teeth are longer or that you have receding gums? (Q2.5) and have you lost teeth in recent years because of mobility? (Q2.11) were significantly associated with moderate/severe periodontitis. Also, for the IWCPDC classification, the question do your gums usually bleed either when brushing or chewing? (Q2.12) and for the CDC-AAP classification, in the last years have you noticed that you see the roots of several of your teeth? (Q2.6) were significantly associated to moderate/severe periodontitis (Table 4). These questions were combined to adjust different multivariable logistic models to estimate the best-reduced model associated with the severity of periodontal disease. From the twelve adjusted models, eight had a sensitivity $>80 \%$. The model with the highest sensitivity (92.2\%) was the one with the four specific questions included and after adjusting by age, sex, level of education, monthly salary and country of origin it was significantly associated with moderate to severe periodontitis $(\mathrm{OR}=21.395 \%$ CI 5.1-90.1), followed by model 4 and 12 with $90 \%$ of sensitivity (Table 6). Models 2 and 7 were the ones with the better values of both sensitivity and specificity, with the best positive and negative predictive values and also significantly associated with moderate to severe periodontitis (Table 6).

\section{6 | Subgroup analyses}

Subgroup analyses were performed by age ( $<45$ vs. $\geq 45$ years), education (under high school vs. over professional training), monthly salary income (low vs. middle/high) and 
tobacco use (never vs. ex/current smoker) (see Supplementary Tables 1-4, respectively, in online Journal of Periodontology) to explore the performance of the self-reported questionnaire within each different group. For all subgroups, the most sensitive question was In the past years have you noticed that your teeth are longer or that you have receding gums? (Q2.5). Also, the question do you think you might have gum disease? (Q2.1) had higher values of sensitivity in participants $<45$ years and in never smokers. This question also had higher values of sensitivity for both education and monthly salary income subgroups.

\section{4 | DISCUSSION}

Our results provide evidence that self-reported measures for periodontitis could be a feasible and useful tool for screening periodontal disease in Spanish population. The 12 itemquestions were easy to understand, proven by the absence of non-response. Only the first general question do you have periodontal disease? (Q1) was difficult to comprehend by study participants. This may indicate that both patients attending a first visit to dental hospitals and individuals from the general population actually does not know what the medical concept of "periodontal disease" means, reflected by the high percentage of false positive cases $(60.8 \%)$.

The 12-item questionnaire had a useful discriminative capability for both detecting individuals with periodontitis (AUC $=0.8595 \% \mathrm{CI} 0.78-0.92$ ) and its moderate/severe form $(\mathrm{AUC}=0.8695 \% \mathrm{CI} 0.79-0.04),{ }^{15}$ with sensitivity and specificity of $77 \%$ and $74 \%$ and $73 \%$ and $87 \%$, respectively; representing moderate validity. ${ }^{16}$ Furthermore, the combination of three specific questions: in the past years have you noticed that your teeth are longer or that you have receding gums? (Q2.5), have you lost teeth in recent years because of mobility? (Q2.11) and do your gums usually bleed either when brushing or chewing? (Q2.12) had nearly high accuracy ${ }^{15}$ for discriminating periodontitis (AUC $=0.8795 \%$ CI 0.81 0.94 ) and high validity ${ }^{16}$ with a sensitivity value of $90.2 \%$ and strongly associated with periodontal disease after adjusting for confounders. The addition of one more question to this minimum set: in the last years have you noticed that you see the roots of several of your teeth? (Q2.6), had both high accuracy $(\mathrm{AUC}=0.8895 \% \mathrm{CI} 0.81-0.94)$ and validity (sensitivity $=92.2 \%$ ) and was also associated with moderate to severe periodontitis after adjusting by important socio-demographic factors.

Comparing the findings with a previous systematic review, ${ }^{17}$ were they concluded that self-reported painful gums, tooth mobility and people's awareness of periodontal disease were the most efficient type of questions to identify periodontitis; our results suggests that the combination of self-reported questions regarding tooth mobility (Q2.11) and gum migration (Q2.5/Q2.6) have a high performance when compared to different clinical classifications of periodontitis and its severity, with sensitivity and specificity values $>80 \%$, representing high validity. ${ }^{16}$ Contrary, the question about painful gums (Q2.7) was not related to periodontitis in the univariate or multivariable models. Although our self-reported questions regarding patient awareness of periodontal or gum disease (Q1/Q2.1/Q2.2) had moderate to high specificity (66.7\% to $98.4 \%$ ), they had low to moderate sensitivity (38.8\% to $67.6 \%)$ and were not associated with periodontitis or its severity in multivariable models. Luo and $\mathrm{Wu}^{18}$ reported that the rate of self-awareness of periodontitis among US adults was low, showing than in those with a clinical diagnosis of periodontitis, $27 \%$ were aware of their condition. Self-reported measures regarding awareness of periodontal disease requires some medical knowledge to understand the concept of periodontitis at the community leve $\mathrm{l}^{19}$ and is strongly influenced by educational level and other socioeconomic factors, such as access to dental care, which would reflect awareness of their condition. ${ }^{18}$ In that context, self-reported measures for periodontitis are commonly used in epidemiological studies ${ }^{19-25}$ to assess its relationship with other diseases. It may be possible that questions regarding tooth mobility and gum migration would be suitable indicators to assess periodontal disease rather than questions regarding self-awareness of their condition.

\subsection{Strengths and limitations}

Our study has several limitations. The sample size precludes us to create a predictive model to estimate the prevalence of periodontitis and its severity in the population under study. Doing so would result in a non-robust model that could not be validated. Another limitation is the language. Some questions included in our questionnaire are native Spanish and has not being validated in English. The others were adapted from the Spanish version of the eight self-reported oral health questions identified by the CDC surveillance initiative for self-reported measures for periodontitis. ${ }^{4}$ Also, the study population may be not representative from the general population, because half of the sample were patients who attended the HOUB and may represent individuals aware and with severe conditions, so results may be not generalizable. However, prevalence of periodontitis in the total sample was $45.5 \%$, $33.3 \%$ for the 35 to 44 years group and $72 \%$ for adult's $\geq 65$ years, which is similar to the prevalence reported by the National Oral Health Survey in Spain in 2015 (33.8\% and $70.9 \%$, respectively). ${ }^{3}$ The full-mouth periodontal examination in six sites per tooth assures an accurately measure of the periodontal status and an objectively classification of the condition according to three standard criterion for classification. 


\section{5 | CONCLUSIONS}

The use of several self-reported questions could be a useful tool for screening periodontitis in the population; specifically, those related with tooth mobility and gum migration. Questions regarding patient's awareness of periodontal disease could be valid, but it is necessary to reconsider their formulation and wording. The set of four self-reported questions proved to have a good performance in a Spanish sample of adults, with high accuracy and high validity for screening periodontitis and its severity; still, large community-based studies are needed to test its validity and predictive performance in external scenarios.

\section{1 | Practical implications}

The combination of several self-reported questions had both high accuracy and validity for screening periodontal disease in the population under study; specifically those related with tooth mobility and gum migration.

\section{CONFLICT OF INTEREST}

Authors declare no potential conflicts of interest.

\section{FUNDING}

This research did not receive any specific grant from funding agencies in the public, commercial, or not-for-profit sectors.

\section{AUTHOR CONTRIBUTIONS}

Dr. Saka-Herrán contributed with the conception and design, the acquisition, analyses and interpretation of data; drafting the article and revised critically for important intellectual content and for final approval of the version to be published, in agreement to be accountable for all aspects of the work. Dr. Jané-Salas contributed with the conception and design, the analyses and interpretation of data; drafting the article and revised critically for important intellectual content and for final approval of the version to be published, in agreement to be accountable for all aspects of the work. Dr. GonzálezNavarro contributed with the conception and design, analyses and the interpretation of data; revised critically for important intellectual content and for final approval of the version to be published, in agreement to be accountable for all aspects of the work. Dr. Estrugo-Devesa contributed with the conception and design, analyses and the interpretation of data; revised critically for important intellectual content and for final approval of the version to be published, in agreement to be accountable for all aspects of the work. Dr. López-López contributed with the conception and design, the analyses and interpretation of data; drafting the article and revised critically for important intellectual content and for final approval of the version to be published, in agreement to be accountable for all aspects of the work.

\section{REFERENCES}

1. Eke PI, Dye BA, Wei L, et al. Update on prevalence of periodontitis in adults in the United States: NHANES 2009-2012. J Periodontol. 2015;86:611-622.

2. Kassebaum NJ, Bernabé E, Dahiya M, Bhandari B, Murray CJL, Marcenes W. Global burden of severe periodontitis in 1990-2010. J Dent Res. 2014;93:1045-1053.

3. Bravo-Pérez M. Oral health survey in Spain 2015 (in Spanish). RCOE. 2016;21:8-48.

4. Eke PI, Dye BA, Wei L, et al. Self-reported measures for surveillance of periodontitis. J Dent Res. 2013;92:1041-1047.

5. Zhan Y, Holtfreter B, Meisel P, et al. Prediction of periodontal disease: modelling and validation in different general German populations. J Clin Periodontol. 2014;41:224-231.

6. Blicher B, Joshipura K, Eke P. Validation of self-reported periodontal disease: a systematic review. J Dent Res. 2005;84:881-890.

7. Slade GD. Interim analysis of validity of periodontitis screening questions in the Australian population. $J$ Periodontol. 2007;78:1463-1470.

8. Khader Y, Alhabashneh R, Alhersh F. Development and validation of a self-reported periodontal disease measure among Jordanians. Int Dent J. 2015;65:203-210.

9. Bujang MA, Adnan TH. Requirements for minimum sample size for sensitivity and specificity analysis. J Clin Diagnostic Res. 2016;10:YE01-YE06.

10. Chaple AM, Gispert EA. The O'Leary index, a.k.a. the "love" index (in Spanish). Rev Cubana Estomatol. 2019;56:1-5.

11. Sociedad española de periodoncia y osteointegración. Diagnostic and periodontal treatment guide (in Spanish). Available at: https://www.sepa.es/web_update/guia-periodontal/. Accessed February 10, 2020.

12. Tonetti MS, Greenwell H, Kornman KS. Staging and grading of periodontitis: framework and proposal of a new classification and case definition. J Clin Periodontol. 2018;45:S149-S161.

13. Page RC, Eke PI. Case definitions for use in population-based surveillance of periodontitis. J Periodontol. 2007;78:1387-1399.

14. Pérez-Jiménez F, Ros E, Solá R, et al. Advices to help cholesterol control with healthy eating (in Spanish). Clín Investig Arteriosc. 2006; 18:104-110.

15. Swets JA. Measuring the accuracy of diagnostic systems. Science. 1988;240:1285-1293.

16. Nelson DE, Holltzman D, Bolen J, Stanwyck C, Mack K. Reliability and validity of measures from the behavioral risk factor surveillance system (BRFSS). Soz Praventivmed. 2001;46:S3-S42.

17. Abbood HM, Hinz J, Cherukara G, Macfarlane TV. Validity of self-reported periodontal disease: a systematic review and metaanalysis. J Periodontol. 2016;87:1474-1483.

18. Luo H, Wu B. Self-awareness of "gum disease" among US adults. J Public Health Manag Pract. 2017;23:e1-e7.

19. Gonzalez-Navarro B, Pintó-Sala X, Corbella E, et al. Associations between self-reported periodontal disease, assessed using a very short questionnaire, cardiovascular disease events and allcause mortality in a contemporary multi-ethnic population: the multi-ethnic study of atherosclerosis (MESA). Atherosclerosis. 2018;278:110-116 
20. Oliveira LS, Lira-Junior R, Figueredo CM, Gomes MB, Fischer RG. Self-reported periodontitis and complications in type 1 diabetes patients: a Brazilian nationwide survey. Braz Dent J. 2016;27:599603.

21. Miyawaki A, Toyokawa S, Inoue K, Miyoshi Y, Kobayashi Y. Selfreported periodontitis and incident type 2 diabetes among male workers from a 5-year follow-up to MY health up study. PLoS One. 2016;11:1-11.

22. Michaud DS, Kelsey KT, Papathanasiou E, Genco CA, Giovannucci E. Periodontal disease and risk of all cancers among male never smokers: an updated analysis of the health professionals follow-up study. Ann Oncol. 2016;27:941-947.

23. Jimenez M, Hu FB, Marino M, Li Y, Joshipura KJ. Type 2 diabetes mellitus and 20 year incidence of periodontitis and tooth loss. Diabetes Res Clin Pract. 2012;98:494-500.

24. Senba T, Kobayashi Y, Inoue K, et al. The association between self-reported periodontitis and coronary heart diseasefrom MY health up study. J Occup Health. 2008;50:283287.
25. Oluwagbemigun K, Dietrich T, Pischon N, Bergmann M, Boeing H. Association between number of teeth and chronic systemic diseases: a cohort study followed for 13 years. PLoS One. 2015;10:1-14.

\section{SUPPORTING INFORMATION}

Additional supporting information may be found online in the Supporting Information section at the end of the article.

How to cite this article: Saka-Herrán C, Jané-Salas E, González-Navarro B, Estrugo-Devesa A, LópezLópez J. Validity of a self-reported questionnaire for periodontitis in a Spanish population. J Periodontol. 2020;91:1027-1038. https://doi.org/10.1002/ JPER.19-0604 